Proceedings

\title{
Multidimensional health inequality among children in Maha- rashtra amid the Covid-19 pandemic
}

Rekha V 1,*

Publisher's Note: MDPI stays neutral with regard to jurisdictional claims in published maps and institutional affiliations.

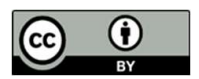

Copyright: (c) 2020 by the authors. Submitted for possible open access publication under the terms and conditions of the Creative Commons Attribution (CC BY) license (http://creativecommons.org/licenses/by/4.0/).
1 Doctoral Candidate, Indian Institute of Technology Bombay, India; Assistant Professor, Government College Munnar, Kerala; rekhapriyav13@gmail.com

* Correspondence: rekhapriyav13@@gmail.com

\begin{abstract}
The study utilises the fourth round of District Level Health Survey to provide districtlevel estimates of the inequalities in multidimensional health deprivations among children in Maharashtra. Multidimensional Health Poverty Index (MHPI), concentration index, geographical maps, and logistic regression model are used to analyse the data at the multiple levels of state, districts, rural-urban residence and gender. The findings indicate that multidimensional health inequalities manifest primarily among the weaker sections of society. The MHPI of under-five children is 0.297 with 46.8 percent of them identified as multidimensionally health poor and experiencing an average of $63.4 \%$ of weighted deprivations. The rural areas are home to a much larger number of multidimensional health poor than urban ones. The spatial profiling shows that worst performers are mostly clustered in and around Marathwada region which is one of the backward areas of Maharashtra. The main predictors of multidimensional health poverty among under-five children are low asset index, educational and employment status of the parents and health conditions of the mother. Recognising the regional heterogeneity in the incidence of multidimensional health poverty and distribution calls for strengthening COVID-19 intervention strategies attuned for specific district targets.
\end{abstract}

Keywords: anthropometry; child health; Covid-19; concentration index; health inequality; Maharashtra; multidimensional health poverty; undernutrition

\section{Introduction}

Maharashtra accounts for the highest number of Covid-19 cases in India. The pandemic and the subsequent lockdown have exacerbated the malnutrition crisis in the state. Many districts in the state are fighting a dual battle of COVID-19 and undernutrition. The numbers are alarming, given that the situation will aggravate further in the absence of social protection schemes. Even though children in the state have shown resilience against the SARS-CoV-2 than adults, pre-existing morbidities and undernutrition pose an enormous risk. Children with multiple anthropometric failures are significantly more likely to experience respiratory infections and fall prey to death. In India, there is significant evidence routinely available on the nutrition profiles of the children. However, health inequality focusing on the regional disparity of child nutrition appears to be reasonably limited in the state. The present study provides district-level estimates of prevalence rates and inequalities in multidimensional health poor children in Maharashtra. Understanding the economic and spatial variation in child health across the district population is paramount, considering the decentralised nature of health delivery platforms. Given the scenario, it is crucial to strengthen COVID-19 intervention strategies through regional and cluster mapping to identify the most disadvantaged children. The main objectives of the study are as follows: The first is to propose a multidimensional approach using AlkireFoster method to measure the complex and multifaceted nature of early childhood health. 
The second objective is to provide district-level estimates of the inequalities in multidimensional health deprivations among children in Maharashtra. The third objective consists of investigating the main determinants of multidimensional health poverty among children. The study also aims to discuss the implications of the pandemic on childhood undernutrition.

\section{Materials and Methods}

The study utilises data from the fourth round of District Level Health Survey to measure the multidimensional nutritional status of under-five children. Multidimensional Health Poverty Index, Concentration Index, geographical maps, and logistic regression model are used to analyse the data at the multiple levels of state, districts, rural-urban residence and gender. Several socio-economic, demographic factors such as wealth quintiles, social background, safe drinking water, insurance, gender and age of the child, education and employment status of the caregiver, BMI and anaemic status of the mother, administrative divisions are included as predictor variables in the study. Among the supply-side variables health providers like ASHA, Anganwadi workers, distance to primary health centre and private hospital are taken as explanatory variables.

Child population is classified as stunted, wasted and underweight if their height-forage, weight-for-height and weight-for-age are below-2 standard deviations from the median of the WHO child growth standards. Children of six months and above are classified as anaemic if the haemoglobin concentration in them is found to be lower than $11.0 \mathrm{~g} / \mathrm{dl}$. The selected indicators capture distinct biological aspects of undernutrition. The stunting measures chronic undernutrition which reflects cumulative deficiencies in growth linked with long term inadequate protein intake and frequent infections. The wasting measures acute undernutrition, usually results from starvation and/or disease. The underweight is a composite indicator which reflects both chronic and acute undernutrition. The anaemia demonstrates micronutrient deficiencies. It has been argued that conventional indicators used on their own cannot truly reflect the overall burden of undernutrition (Svedberg, 2000; Nandy et al. 2005; Nandy and Miranda, 2008). Drawing insights from Alkire and Foster all the four child nutritional outcomes are aggregated into one single composite index called Multidimensional Health Poverty Index (MHPI).

\subsection{Multidimensional Health Poverty Index}

A child is considered multidimensionally health poor if he or she is impoverished in two out of the four malnutrition indicators that are included in the index. An MHPI value of 1 indicates the highest health deprivation level when the child is deprived in four indicators, while 0 indicates the lowest when the child is not deprived in any of the four indicators. Multidimensional Health Poverty Index consists of three interrelated measures. Headcount ratio $(\mathrm{H})$ or incidence of multidimensional poverty indicates the proportion of children who are simultaneously stunted, wasted, underweight and anaemic. The intensity of deprivation (A) also called the breadth of multidimensional deprivation is the average percentage of impoverishments among the health poor. Adjusted Headcount ratio $\left(\mathrm{M}_{0}\right)$ also known as Multidimensional Health Poverty Index is defined as the product of a multidimensional headcount (prevalence of health deprivations) and the average intensity of the health deprived poor. The MHPI is used as the binary dependent variable to provide a comprehensive picture of the extent and nature of multidimensional inequalities among children in Maharashtra.

\subsection{Concentration Index}

Concentration curve and Concentration index $(\mathrm{CI})$ have been extensively used in the literature to measure socio-economic inequality in any health outcome []. (Wagstaff, Paci \& van Doorslaer 1991; Cuyler \&Wagstaff 1993; Kakwani, Wagstaff \& van Doorslaer 1997). The concentration curve plots the cumulative proportions of multidimensionally health 
poor children on the y-axis against the cumulative population shares ranked by wealth index of living standards on the x-axis. The $\mathrm{CI}$ is defined as twice the area between the concentration curve and the egalitarian line (the 45-degree line).

$$
C=\frac{2}{\mu} \operatorname{cov}(y, r)
$$

where $C$ is the concentration index, $y$ is the health variable i.e., MHPI, $\mu$ is its mean and $r$ is the relative fractional rank of the individual in the socioeconomic distribution.

\section{Results and Discussion}

Table 1 shows that MHPI of under-five children is 0.297 with 46.8 percent of them identified as multidimensionally health poor and experiencing an average of $63.4 \%$ of weighted deprivations. This means that health poor children in Maharashtra experience $29.7 \%$ of the deprivations that would be experienced if every child in Maharashtra was malnourished based on all the four indicators. Out of it, 48.9 percent and 43.9 percent of multidimensional health poor children are from rural and urban regions respectively. In Maharashtra, 47.2 and 46.4 percent of below-five boys and girls are MHPI poor since they are deprived in two or more of the weighted malnutrition indicators. The depth of the deprivations is around $63 \%$ for both the gender which means that malnourished children in Maharashtra, on average experience $63 \%$ of the weighted deprivations.

Table 1. Multidimensional Health Poverty Indices and CI values across regions of Maharashtra.

\begin{tabular}{lcccc}
\hline \multicolumn{1}{c}{ Subgroups/Indices } & $\mathbf{H}^{\mathbf{1}}$ & $\mathbf{A}^{\mathbf{1}}$ & MHPI & $\mathbf{C I}^{\mathbf{1}}$ \\
\hline Boys & 47.2 & 63.5 & 0.300 & -0.178 \\
Girls & 46.4 & 63.3 & 0.294 & -0.143 \\
Rural & 48.9 & 64.2 & 0.314 & -0.151 \\
Urban & 43.9 & 62.1 & 0.273 & -0.154 \\
Amravati & 41.6 & 62.8 & 0.261 & -0.099 \\
Aurangabad & 53.8 & 65.2 & 0.351 & -0.174 \\
Nashik & 51.8 & 61.9 & 0.321 & -0.102 \\
Nagpur & 40.9 & 62.7 & 0.257 & -0.138 \\
Pune & 40.2 & 62.9 & 0.253 & -0.223 \\
Konkan & 40.7 & 62.9 & 0.256 & -0.189 \\
& & & & \\
Total & 46.8 & 63.4 & 0.297 & -0.161 \\
\hline
\end{tabular}

${ }^{1}$ H-Multidimensional Headcount, A-Intensity of Deprivations, CI-Concentration Index.

\subsection{District-level Health Poverty Estimates}

District-wise analysis exhibits that Nanded, a district located on the central west part of Maharashtra is the poorest health deprived district having 60.3 percent of health poor followed by Nandurbar (58.1\%) and Buldana ( $57.7 \%$ ). On the other hand, the lowest prevalence of multiple health deprived children is found in Pune (34.9\%) and Mumbai city $(35.8 \%)$. The intensity of health deprivations is remarkably higher compared to the headcount ratio of health poor in all the districts of Maharashtra. The spatial profiling (Figure 1) shows that worst performers are mostly clustered in and around Marathwada region which is one of the backward areas of Maharashtra. Thane a tribal district, close to Mumbai which is the epicentre of the pandemic in the country have $48 \%$ of its child population multidimensionally health poor. In Thane, $18.7 \%$ of its children living under severe multiple deprivations, where the intensity of health poverty is at an alarming rate of $70 \%$. Multidimensional health poverty has a remarkably high concentration among the children from poor socio-economic status in the majority of the districts in Maharashtra 


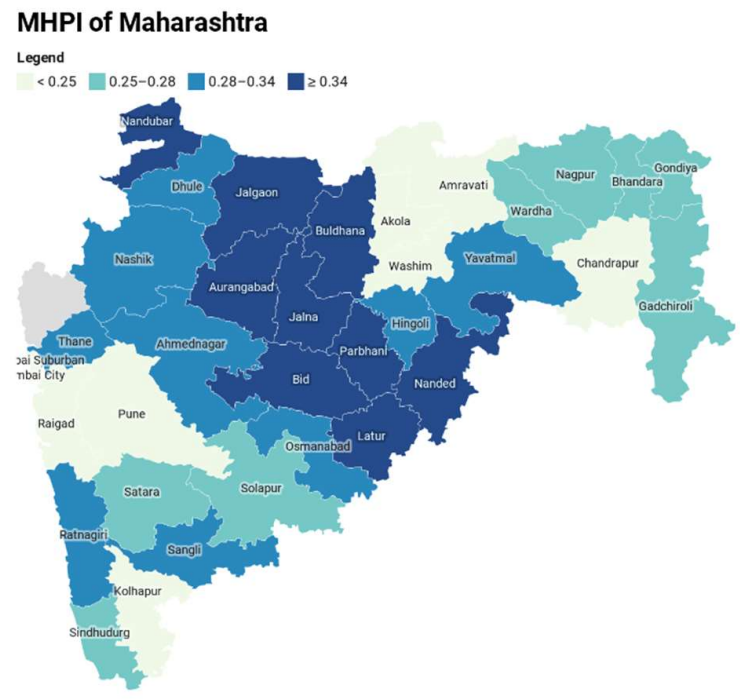

(a)

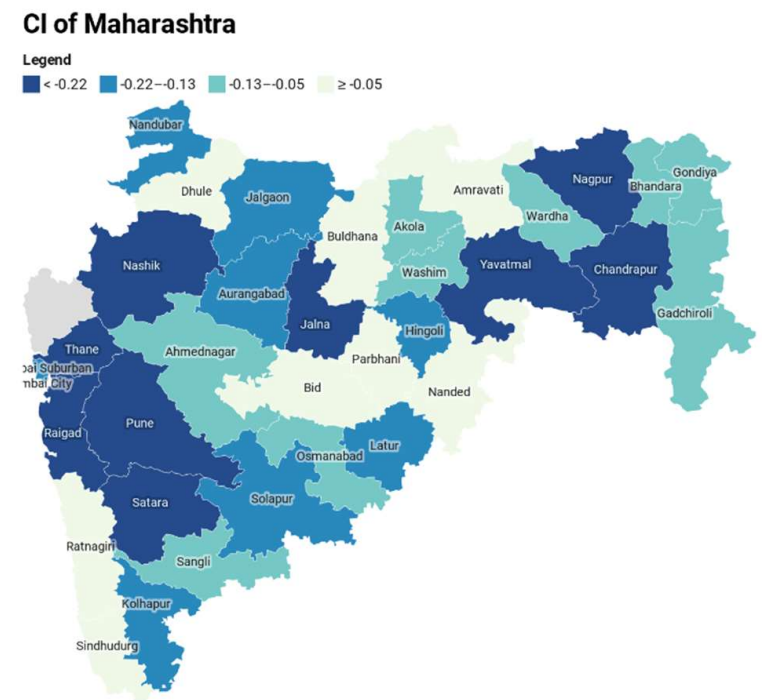

(b)

Figure 1. Spatial pattern in the prevalence of and inequality in the multidimensional health indicators: (a) Districts wise values of Multidimensional Health Poverty Index; (b) Concentration Index (CI) values across different districts of Maharashtra.

\subsection{Multivariate Regression Analysis}

The dependent variable for regression analysis is, if the child is multidimensionally health poor with two or more overlapping nutritional deprivations, assign value one, otherwise zero. Relative to the base category children living in households with less income and being from SC/ST family are more likely to be malnourished in multiple dimensions. Parental education and employment status are found having the strong association with the health poverty status of the children in Maharashtra. Health status of the mother significantly influences the probability of a child being health poor. The estimated marginal effects indicate that having an underweight and anaemic mother increases the likelihood of multidimensional health poverty. The regional location of the households also plays an important role in reducing nutritional outcomes. None of the supply-side variables except Anganwadi workers and private hospitals seem to have significant effect on the multidimensional health poverty among children.

The results from the distributive aspects of multidimensional health poverty indicate that the magnitude of wealth based inequality concerning child malnutrition is higher and manifest primarily among the weaker sections of the society. The issue of inter-district and inter-regional variations in malnutrition and its distribution cannot be dismissed amidst the relative prosperity of the state. Many districts of Maharashtra perform poorly on both accounts of the prevalence and inequalities in chronic malnutrition. The nutrionally backward areas are concerted in the tribal districts of Marathwada division and few from Vidarbha and Amaravati. Inequalities are reported to be lower in these districts. At the other end of the scale are relatively well-off districts with higher inequalities which are mostly from the Western and Konkan divisions. Many districts in Maharashtra have multiple burdens of undernourishment and have high inequalities, while several others have low levels of inequalities in child health. We have seen that several districts have relatively higher inequality levels even at low levels of average malnutrition. This suggests that the decline in average malnutrition rate does not necessarily lead to diminishing inequalities. We need to have policies to address both incidence and inequality issues separately. 
Multidimensional health poverty index encompasses the various forms of undernutrition experienced by the child at the same time. The MHPI illuminates for policy makers who is health poor and how intensely they suffer from health deprivations and allow them to take direct action for the population groups at highest risk. The rural areas are home to a much larger number of multidimensional health poor than urban ones. Gender-wise examination reveals that boys are found to be slightly more multidimensionally health poor than their opposite sex. District level health poverty estimates reveal geographical clustering of multidimensional health poor. At least, every two children on average, is multidimensionally health poor in 18 districts of Maharashtra which accounts for the majority of the population. The results of this study offer critical implications for anti-malnutrition programme. Social protection schemes should focus the priority areas and maintain the steady supply of essential commodities to poorest households. Investments need to be done in maternal nutrition monitoring and surveillance through pregnancy, infancy and childhood to break intergenerational transmission of health.

Funding: This research received no external funding

Conflicts of Interest: The authors declare no conflict of interest

\section{References}

1. Alkire, S., \& Foster, J. (2011). Understandings and misunderstandings of multidimensional poverty measurement. The Journal of Economic Inequality, 9(2), 289-314.

2. Alkire, S., Foster, J. E., Seth, S., Santos, M. E., Roche, J., \& Ballon, P. (2015). Multidimensional poverty measurement and analysis: Chapter 5-the Alkire-Foster counting methodology.

3. Alkire, S., \& Foster, J. (2011). Counting and multidimensional poverty measurement. Journal of public economics, 95(7-8), 476-487.

4. Nandy, S., \& Svedberg, P. (2012). The Composite Index of Anthropometric Failure (CIAF): An alternative indicator for malnutrition in young children. In Handbook of anthropometry (pp. 127-137). Springer, New York, NY.

5. Wagstaff A., Paci P., van Doorslaer E.(1991).On the Measurement of Inequalities in Health. Social Science and Medicine .33(5) .pp.545-57.

6. Wagstaff, A., Van Doorslaer, E., Watanabe, N. (2003). On decomposing the causes of health sector inequalities with an application to malnutrition inequalities in Vietnam. Journal of Econometrics, 112(1), 207-223.

7. Wagstaff, A., Watanabe, N. (2000). Socioeconomic inequalities in child malnutrition in the developing world (Vol. 2434). World Bank, Development Research Group, Poverty and Human Resources, and, Human Development Network, Health, Nutrition, and Population Team. 